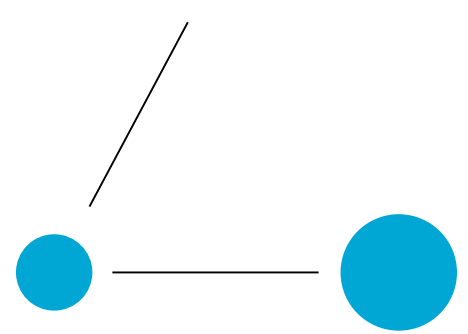

A N N A L E S HENRI LEBESGUE

JASON STARR

ABstraCt. - For a complex projective manifold that is rationally connected, resp. rationally simply connected, every finite subset is connected by a rational curve, resp. the spaces parameterizing these connecting rational curves are themselves rationally connected. We prove that a projective scheme over a global function field has a rational point if it deforms to a rationally simply connected variety in characteristic 0 with vanishing elementary obstruction. This gives new, uniform proofs over these fields of the Period-Index Theorem, the quasi-split case of Serre's "Conjecture II", and Lang's $C_{2}$ property.

Keywords: rationally simply connected varieties, rational points, degenerations. 2020 Mathematics Subject Classification: 14G05, 14M22, $14 \mathrm{D} 06$.

DOI: https://doi.org/10.5802/ahl.65

(*) JMS was supported by NSF Grants DMS-0846972 and DMS-1405709, as well as a Simons Foundation Fellowship. CX was supported by the National Science Fund for Distinguished Young Scholars (11425101), "Algebraic Geometry". 
RÉSUMÉ. - Si une variété projective complexe est rationnellement connexe, chaque ensemble fini de points est contenu dans une courbe rationnelle ; si elle est rationnellement simplement connexe, les espaces paramétrant ces courbes rationnelles sont eux-mêmes rationnellement connexes. Nous montrons qu'un schéma projectif sur un corps global de caractéristique non nulle possède un point rationnel s'il se déforme en une variété rationnellement simplement connexe de caractéristique zéro dont l'obstruction élémentaire s'évanouit. Pour de tels corps, on obtient ainsi des preuves uniformes du théorème période-indice, du cas quasi-déployé de la « Conjecture II » de Serre, et de la propriété $C_{2}$ de Lang.

\section{Statement of Results}

For a field $F$ and a projective $F$-scheme $X_{F}$, does $X_{F}$ have an $F$-rational point? Colliot-Thélène and Sansuc defined an obstruction to existence of an $F$-rational point, the elementary obstruction, [CTS87, Section 2.2]. If $X_{F} \times_{\operatorname{Spec} F} \operatorname{Spec} \bar{F}$ is integral and smooth, the elementary obstruction vanishes if and only if $X_{F}$ admits a universal torsor, cf. [CTS87, Proposition 2.2.3], [Sko01, Theorem 2.3.4]. For geometrically integral and smooth $X_{F}$, the universal torsor is a torsor over $X_{F}$ for a multiplicative group $F$-scheme $Q$ such that for every field extension $E / F$, for every multiplicative group $F$-scheme $R$, every $R$-torsor over $X_{F} \times_{\operatorname{Spec} F} \operatorname{Spec} E$ arises from the universal torsor by a unique morphism of group $E$-schemes, $Q \times_{\operatorname{Spec} F} \operatorname{Spec} E \rightarrow R$. For our geometric applications, it is important to extend these notions to a flat, projective scheme over a base scheme rather than a base field. This has been developed in [Pir12, Definition, p. 468] and in [Zhu19]. We review below the constructions from [Zhu19].

When is vanishing of the elementary obstruction a sufficient condition for existence of an $F$-rational point? Let $\mathbb{F}(\eta)$ be a global function field: the function field of a smooth projective curve over a finite field $\mathbb{F}$. For a projective $\mathbb{F}(\eta)$-variety $f_{\eta}: X_{\eta} \rightarrow$ Spec $\mathbb{F}(\eta)$, our main theorem gives an $\mathbb{F}(\eta)$-rational point of $X_{\eta}$ provided that there exists a lift to characteristic 0 of a projective model that has vanishing elementary obstruction and that is a rationally simply connected fibration. A key role is played by Esnault's theory of rational points of specializations over finite fields of varieties of coniveau $\geqslant 1$, specifically [Esn07, Corollary 1.2].

\subsection{Rationally simply connected fibration}

Let us first fix some notation: Our main theorem will be for degenerations over $\mathbb{F}(\eta)$ that can be lift to rationally simply connected varieties over a field of characteristic 0 , which is the function field of a curve $C_{K}$ over a field $K=K(R)$ where $R$ is a Henselian discrete valuation ring (DVR) (see [Gre66] for the definition and examples of Henselian DVR) with residue field $\mathbb{F}$.

The following is a precise definition of lifts to characteristic 0 .

Definition 1.1. - A projective extension over $R$ of $X_{\eta}$ is a surjective morphism,

$$
f_{R}: X_{R} \rightarrow C_{R},
$$

between projective, flat, $R$-schemes satisfying the following. 
(1) The morphism $C_{R} \rightarrow$ Spec $R$ has connected geometric fibers.

(2) The $R$-smooth locus $C_{R \text {, sm }}$ of $C_{R}$ contains both the generic fiber $C_{K}$ and one of the generic points $\eta$ of the closed fiber $C_{\mathbb{F}}$ whose residue field equals $\mathbb{F}(\eta)$.

(3) The flat locus in $C_{R}$ of the morphism $f_{R}$ contains both the generic fiber $C_{K}$ and $\eta$.

(4) The fiber of $f_{R}$ over $\eta$ equals $f_{\eta}$.

Now, we explain what we mean by simply rationally connected fibrations. While a rationally connected variety $X$ being rationally simply connected roughly means the space of rational curves on $X$ is rationally connected, to give a precise definition is a lot more technical. For our purpose in this note, we will consider two cases that the "rational simple connectedness" has been confirmed.

Hypothesis 1.2 ([dJHS11]). - Let $\mathcal{L}_{K}$ be an $f_{K}$-ample line bundle on $\frac{X_{K}}{C_{K}}$. We assume the following.

(1) There exists an open subset $C_{K}^{\circ} \subset C_{K}$ and an open set $U$ of $X^{\circ}:=X \times_{C_{K}} C_{K}^{\circ}$ surjecting to $C^{\circ}$ such that the geometric fibres of the evaluation morphism from the Kontsevich moduli space with the vertical curve classes intersection $\mathcal{L}$ being 1

$$
\text { ev: } \overline{\mathcal{M}}_{0,1}\left(\frac{X_{K}^{\circ}}{C_{K}^{\circ}}, 1\right) \rightarrow X_{K}^{\circ}
$$

over $U$ are nonempty, irreducible and rationally connected.

(2) There exists a positive integer $m_{0}$ and an open subset $V$ of $X_{K}^{\circ} \times_{C_{K}^{\circ}} X_{K}^{\circ}$ surjecting to $C_{K}^{\circ}$ and such that the geometric fibres of the evaluation morphism (See [dJHS11, P. 30])

$$
\operatorname{ev}_{1, m_{0}+1}: \operatorname{FreeChain}_{2}\left(\frac{X_{K}^{\circ}}{C_{K}^{\circ}}, m_{0}\right) \rightarrow X_{K}^{\circ} \times_{C_{K}^{\circ}} X_{K}^{\circ}
$$

over $V$ are nonempty, irreducible and birationally rationally connected.

(3) There exists a geometric fibre of $X_{K} \rightarrow C_{K}$ which has a very twisting scroll (see [dJHS11, Definition 12.3]).

The above Definition 1.1 can be applied to fibrations of homogeneous spaces with Picard number 1, see [dJHS11, Section 15]. In [Zhu19], the setting is extended to fibrations of homogeneous spaces with general Picard number in the following way.

Hypothesis 1.3 ([Zhu19, 5.1]). - Let $f_{K}: X_{K} \rightarrow C_{K}$ be a smooth family of projective homogeneous spaces. Assume that the relative Picard number, i.e., the rank of $\mathrm{Pic}_{X_{K} / C_{K}}\left(C_{K}\right)$ is one. Assume that the Picard number of the geometric generic fiber of $f_{K}$ is $r$. Let $S$ be the character $C_{K}$-group scheme of $\mathrm{Pic}_{X_{K} / C_{K}}$. Assume that the relative universal $S$-torsor $T$ exists for the family.

\subsubsection{Main Theorem}

The main theorem is as follows. 
TheOREm 1.4. - For every projective $\mathbb{F}(\eta)$-scheme $X_{\eta}$, for every projective extension over $R$ of $X_{\eta}$, if the generic fiber of $f_{K}$ has vanishing elementary obstruction, [CTS87, Section 2.2], and if $f_{K}$ is either a rationally simply connected fibration as in Hypothesis 1.2 or a Homogeneous spaces fibration satisfying Hypothesis 1.3, then $X_{\eta}$ has an $\mathbb{F}(\eta)$-point.

Remark 1.5. - One main application of the theory of rationally simply connected varieties is that one can extend various results on the existence of rational points on rationally connected varieties over certain $C_{1}$ fields, to rationally simply connected varieties over $C_{2}$ fields obtained by adding one variable.

Theorem 1.4 can be considered to roughly say that for $\mathbb{F}(\eta)$, the degenerations of simply rationally connected varieties also has a rational point. The proof will use a similar result for degenerations of rationally connected varieties over $\mathbb{F}$ (see [Esn07, EX09] or Section 2.2). In characteristic 0, there is indeed a purely geometric explanation on why in terms of the existence of rational points, the degeneration of rationally connected varieties admit the same property as rationally connected varieties, see [HX09].

The corollaries of Theorem 1.4 include several well-known existence theorems for rational points, as well as some new consequences.

Corollary 1.6. - [Lan52, Corollary, p. 378] Every intersection $X_{\eta, 1} \cap \ldots \cap X_{\eta, b}$ in $\mathbb{P}_{\mathbb{F}(\eta)}^{n}$ of hypersurfaces of degrees $\left(d_{1}, \ldots, d_{b}\right)$ satisfying $d_{1}^{2}+\ldots+d_{b}^{2} \leqslant n$ has an $\mathbb{F}(\eta)$-point.

Corollary 1.7 (Period-Index Theorem). - Every central simple algebra over $\mathbb{F}(\eta)$ has period equal to the index. This also follows from the Brauer-Hasse-Noether theorem and Hasse's Global Structure Theorem over $\mathbb{F}(\eta)$, cf. [Roq05, pp. 40-41].

Corollary 1.8. - [Har75] For every simply connected, semisimple group $\mathbb{F}(\eta)$-scheme $G$ that is quasi-split, every $G$-torsor over $\mathbb{F}(\eta)$ has an $\mathbb{F}(\eta)$-point, i.e., the quasi-split case of Serre's "Conjecture II" holds for $\mathbb{F}(\eta)$.

One new consequence follows from Theorem 1.4 and Robert Findley's work on rational simple connectedness of hypersurfaces in Grassmannians, [Fin10, Theorem 1.8]. Let $\ell>0$ and $m \geqslant 2 \ell$ be integers. Denote by $G_{\ell, m}$ the Grassmannian scheme over $\mathbb{F}(\eta)$ parameterizing $\ell$-dimensional quotient vector spaces of a fixed vector space of dimension $m$ (up to replacing $\ell$ by $m-\ell$, we can always assume that $m \geqslant 2 \ell$ ).

Corollary 1.9. - Let $\ell>0$ and $m \geqslant 2 \ell$ be integers. Let $\mathcal{G}$ be an $\mathbb{F}(\eta)$-scheme whose elementary obstruction vanishes and with $\mathcal{G} \times_{\operatorname{Spec}} \mathbb{F}(\eta)$ Spec $\overline{\mathbb{F}(\eta)}$ isomorphic to $G_{\ell, m} \times_{\operatorname{Spec} \mathbb{F}(\eta)} \operatorname{Spec} \overline{\mathbb{F}(\eta)}$. Every hypersurface in $\mathcal{G}$ of Plücker degree $d$ satisfying $(3 \ell-1) d^{2}-d<m-4 \ell-1$ has an $\mathbb{F}(\eta)$-point.

Unfortunately, the above bound is likely not the optimal one. For instance, it does not specialise to the bound in Corollary 1.6 when $G_{\ell, m}$ is a projective space.

\section{The Proof of the Main Theorem}

In this section we review Esnault's theory, [Esn07], we review the constructions of Abel sequences from [dJHS11] and [Zhu19], and we prove Theorem 1.4. In the next 
section, we review the technique of discriminant avoidance from [SdJ10] and [dJHS11, Section 16], and we prove the corollaries. The proof of Theorem 1.4 has three components. The main new component is the use of Esnault's Theorem, [Esn07, Corollary 1.2].

\subsection{Review of Esnault's Theorem.}

As above, let $R$ be a Henselian DVR with finite residue field $\mathbb{F}$ and with fraction field $K$. For the following discussion, we do not assume that $K$ has characteristic 0 , although in applications $K$ does have characteristic 0 . Let $\widetilde{Z}_{R} \rightarrow$ Spec $R$ be a projective, flat morphism such that the generic fiber $\widetilde{Z}_{K}$ is smooth and geometrically connected over $K$. The following Theorem 2.1 was first proved in mixed characteristic in [Esn07]. The equicharacteristic result was proved in [EX09].

Theorem 2.1. - [Esn07, Theorem 1.1] [EX09, Theorem 1.1] Assume that the $\ell$-adic cohomology of the geometric generic fiber $H^{i}\left(X_{K} \times_{\operatorname{Spec} K} \operatorname{Spec} \bar{K}\right)$ is supported in codimension $\geqslant 1$ for all $i \geqslant 1$. There exists a surjective, projective morphism $\mathcal{Z}_{R} \rightarrow \widetilde{Z}_{R}$ such that the set of $\mathbb{F}$-rational points of the closed fiber $\mathcal{Z}_{\mathbb{F}}$ is congruent to 1 modulo $q=|\mathbb{F}|$.

Recall that the $\ell$-adic cohomology $H^{i}\left(\widetilde{Z}_{K} \otimes_{\operatorname{Spec} K} \operatorname{Spec} \bar{K}\right)$ is supported in codimension $\geqslant 1$ if for every $\alpha$ in the cohomology group, there exists a closed subset of codimension $\geqslant 1, D \subset \widetilde{Z}_{K}$, such that the restriction of $\alpha$ vanishes in the cohomology group $H^{i}\left((\widetilde{Z} \backslash D) \times_{\operatorname{Spec} K} \operatorname{Spec} \bar{K}\right)$. Via the technique of Bloch-Srinivas, Esnault proved, [Esn03], that whenever $\widetilde{Z}_{K} \times_{\text {Spec } K} \operatorname{Spec} \bar{K}$ is rationally chain connected, then for every $i \geqslant 1$, the cohomology is supported in codimension $\geqslant 1$, and thus Theorem 2.1 applies. A smooth, projective variety $\widetilde{Z}_{K}$ over $K$ is rationally chain connected if for every algebraically closed field extension $k / K$, for every pair of $k$-points of $\widetilde{Z}_{K}$ there exists a connected, finite union of images of $k$-morphisms from $\mathbb{P}_{k}^{1}$ to the variety such that both $k$-points are contained in the union. If $K$ has characteristic 0 , then $\widetilde{Z}_{K}$ is rationally chain connected if and only if $\widetilde{Z}_{K}$ is rationally connected, i.e., for every pair of $k$-points, there exists a single $k$-morphism from $\mathbb{P}_{k}^{1}$ to the variety whose image contains both $k$-points.

The proof of Theorem 2.1 is a combination of several techniques. The authors encourage readers to refer to the original articles [Esn07, EX09], but we summarize the method. The proof is more direct when $K$ has characteristic 0 , as suffices for this article. First, by de Jong's alteration of singularities, [dJ97], there exists a birational, projective morphism $\mathcal{Z}_{R} \rightarrow \widetilde{Z}_{R}$ of flat $R$-schemes such that $\mathcal{Z}_{R}$ is the geometric quotient of a finite group $\Gamma$ acting by $R$-automorphisms on a flat, projective $R$-scheme $\mathcal{W}_{R}$ that is regular. In particular, if $K$ has characteristic 0 (the case of interest in what follows), the cohomology $H^{i}\left(\mathcal{Z}_{K} \times_{\operatorname{Spec} K} \operatorname{Spec} \bar{K}\right)$ equals the $\Gamma$-invariants $H^{i}\left(\mathcal{W}_{K} \times_{\text {Spec } K} \operatorname{Spec} \bar{K}\right)^{\Gamma}$. Using Esnault's earlier work, [Esn06], and Gabber's purity theorem, cf. [Fuj02], it follows that for every $i \geqslant 1$, the eigenvalues of the geometric Frobenius acting on $H^{i}\left(\mathcal{Z}_{K} \times_{\text {Spec } K}\right.$ Spec $\left.K^{u}\right)$ and on the relative cohomology group $H_{\mathcal{Z}_{\overline{\mathbb{F}}}}^{i}\left(\mathcal{Z}_{K} \times_{\text {Spec } R} R^{u}\right)$ are algebraic integers divisible by $q$. Here $R^{u}$ denotes the maximal 
unramified extension of $\bar{k}$ (usually the ring of Witt vectors of $\overline{\mathbb{F}}$ ) and $K^{u}$ is the fraction field of $R^{u}$. Finally, the relative cohomology exact sequence implies that for every $i \geqslant 1$, the eigenvalues of the geometric Frobenius acting on $H^{i}\left(\mathcal{Z}_{\overline{\mathbb{F}}}\right)$ are algebraic integers divisible by $q$. By the Lefschetz trace formula, [Jou77, Corollary III.4.8, p. 109], this implies the congruence of the number of $\mathbb{F}$-points of $\mathcal{Z}_{\mathbb{F}}$. In particular, $\mathcal{Z}_{\mathbb{F}}$ has at least one $\mathbb{F}$-point. Since there is an $\mathbb{F}$-morphism from $\mathcal{Z}_{\mathbb{F}}$ to $\widetilde{Z}_{\mathbb{F}}$, it follows that $\widetilde{Z}_{\mathbb{F}}$ has at least one $\mathbb{F}$-point (without stronger hypotheses, however, we lose the more refined congruence for the number of $\mathbb{F}$-points modulo $q$ ).

\subsection{Application of Esnault's Theorem to the Hilbert scheme.}

We apply Theorem 2.1 to a scheme obtained as a blowing up of a closed subscheme of the relative Hilbert scheme of $X_{R}$ over Spec $R$. Since $f_{R}$ is projective and since $C_{R}$ is projective over Spec $R$, the relative Hilbert scheme Hilb $X_{R} / R$ has connected components that are projective over $\operatorname{Spec} R$. There is an open subscheme $\operatorname{Sec}\left(X_{R} / C_{R} / R\right)$ of Hilb $X_{R} / R$ parameterizing closed subschemes of $X_{R}$ arising as closed images of sections of $f_{R}$, cf. [Gro62, Part IV.4.c, p. 221-219]. Denote by $\Sigma\left(X_{R} / C_{R} / R\right)$ the closure in Hilb $X_{R} / R$ of this open subscheme. A smooth pseudosection of $\operatorname{Sec}\left(X_{K} / C_{K} / K\right)$ is a projective $K$-morphism $\nu_{K}: \widetilde{Z}_{K, I} \rightarrow \Sigma\left(X_{K} / C_{K} / K\right)$ pulling back $\operatorname{Sec}\left(X_{K} / C_{K} / K\right)$ to a dense open subscheme of $\widetilde{Z}_{K, I}$ and such that $\widetilde{Z}_{K, I}$ is a smooth, projective $K$-scheme whose base change to Spec $\bar{K}$ is rationally connected, cf. [GHMS05, Definition 1.2]. Esnault's Theorem reduces Theorem 1.4 to existence of a smooth pseudosection.

Proposition 2.2. - For every projective $\mathbb{F}(\eta)$-scheme $X_{\eta}$, for every projective extension over $R$ of $X_{\eta}$, for every smooth pseudosection of $\operatorname{Sec}\left(X_{K} / C_{K} / K\right)$, there exists an $\mathbb{F}(\eta)$-point of $X_{\eta}$, a finite flat extension of DVRs $R \rightarrow R^{\prime}$ with fraction field extension $K \rightarrow K^{\prime}$, and a $K^{\prime}$-point of the pseudosection parameterizing a section $\sigma_{K^{\prime}}$ whose specialization over $\eta \times_{\text {Spec } R}$ Spec $R^{\prime}$ equals the base change of the $\mathbb{F}(\eta)$-point of $X_{\eta}$.

Proof. - By taking closures in an appropriate projective space, there exists a flat, projective $R$-scheme $\widetilde{Z}_{R, I}$ and an $R$-morphism $\nu_{R}: \widetilde{Z}_{R, I} \rightarrow$ Hilb $_{X_{R} / R}$ whose $K$-fiber is $\nu_{K}$. By Theorem 2.1 for the characteristic 0 field $K$, there exists an $\mathbb{F}$-rational point of $\widetilde{Z}_{\mathbb{F}, I}$ mapping to an $\mathbb{F}$-rational point of $\Sigma\left(X_{R} / C_{R} / R\right)$ corresponding to a closed subscheme $\mathcal{C}_{\mathbb{F}}$ of $X_{\mathbb{F}}$.

Since $\Sigma\left(X_{R} / C_{R} / R\right)$ is the closure of $\operatorname{Sec}\left(X_{R} / C_{R} / R\right)$, there exists a finite flat extension of DVRs, $R \rightarrow R^{\prime}$, and a lifting of the $\mathbb{F}$-point to an $R^{\prime}$-point of $\Sigma\left(X_{R} / C_{R} / R\right)$ whose generic point, $\operatorname{Spec} K^{\prime}$, maps to $\operatorname{Sec}\left(X_{R} / C_{R} / R\right)$. This $K^{\prime}$-point parameterizes the closed image of a section $\sigma_{K^{\prime}}$ of $f_{K^{\prime}}: X_{K^{\prime}} \rightarrow C_{K^{\prime}}$.

Since $X_{R^{\prime}} \rightarrow C_{R^{\prime}}$ is proper, by the valuative criterion of properness, the rational section $\sigma_{K^{\prime}}$ extends to every codimension one point of the normal locus of $C_{R^{\prime}}$. By Item (2) of Definition 1.1, every point of $C_{R^{\prime}}$ lying over $\eta$ is a codimension one point at which $C_{R^{\prime}} \rightarrow \operatorname{Spec} R^{\prime}$ is smooth, and hence at which $C_{R^{\prime}}$ is normal. Thus, the closure of the image of $\sigma_{K^{\prime}}$ in $X_{K^{\prime}}$ restricts over $\eta$ to the closed image of a rational section. Since this closed subset is the base change of $\mathcal{C}_{\mathbb{F}}$, also the restriction 
of $\mathcal{C}_{\mathbb{F}}$ over $\eta$ is the closed image of a rational section. This rational section is an $\mathbb{F}(\eta)$-rational point of $X_{\eta}$.

\subsection{The associated Abelian variety, Abel torsors, and Abel maps.}

In order to apply Proposition 2.2, we need closed subschemes that are potentially equal to pseudosections. Every morphism from a pseudosection to a torsor for an Abelian $K$-variety is constant. Thus, every pseudosection must be contained in a fiber for every morphism from the Hilbert scheme to a torsor for an Abelian $K$ variety. The open subscheme $\operatorname{Sec}\left(X_{R} / C_{R} / R\right)$ is contained in the maximal open subscheme Hilb $X_{R} / R$,CM of the Hilbert scheme parameterizing Cohen-Macaulay closed subschemes of pure relative dimension 1 . Thus, every pseudosection also has dense intersection with this open subscheme. So every pseudosection is contained in a fiber of every $K$-morphism from Hilb $_{X_{R} / R}^{0}$ to a torsor for an Abelian $K$-variety. What is the "universal" such morphism? There is a natural answer to this question under the following hypothesis.

Definition 2.3. - [Zhu19, Definition 2.10] For a Noetherian scheme T, a Tscheme $f: X_{T} \rightarrow T$ that is projective locally over $T$ is Picard-free if

(i) $f$ is flat with integral geometric fibers $X_{t}$,

(ii) every $X_{t}$ is locally complete intersection and is smooth in codimension $\leqslant 3$,

(iii) $h^{1}\left(X_{t}, \mathcal{O}_{X_{t}}\right)$ and $h^{2}\left(X_{t}, \mathcal{O}_{X_{t}}\right)$ equal 0 , and

(iv) every finite, Abelian, étale cover of $X_{t}$ is trivial.

The Condition (ii) above implies each finite type closed subscheme of $\mathrm{Pic}_{X_{T} / T}$ is proper over $T$ (see [Gro05, XI, 3.13]).

Remark 2.4. - Assume that $f_{K}$ is Picard-free, e.g., a rationally simply connected fibration also satisfying Condition (ii).

(i) The relative Picard scheme $P=\operatorname{Pic}_{f_{K}}$ over $C_{K}$ is étale locally a constant group scheme $\mathbb{Z}^{\rho}$ in the sense of [DG70, Definition X.5.1], cf. [Zhu19, Proposition 2.9].

(ii) The Cartier dual group scheme $Q=D(P)=\operatorname{Hom}_{C_{K}-\mathrm{gp}}\left(P, \mathbb{G}_{m, C_{K}}\right)$, is étale locally isomorphic to $\mathbb{G}_{m, C_{K}}^{\rho}$, cf. [DG70, Proposition X.5.3].

(iii) The $K$-stack $B Q_{C_{K} / K}$ of $Q$-torsors over $C_{K}$ is a locally finitely presented, algebraic $K$-stack, [Lie06, Proposition 2.3.4], equal to a gerbe over the coarse moduli space $\left|B Q_{C_{K} / K}\right|$, which is a smooth group $K$-scheme whose identity component $\left|B Q_{C_{K} / K}^{0}\right|$ is an Abelian $K$-variety.

(iv) The component group $\Lambda\left(Q / C_{K} / K\right)$ of $\left|B Q_{C_{K} / K}\right|$ is an étale group $K$-scheme whose associated Galois module $\Lambda\left(Q / C_{K} / K\right)(\operatorname{Spec} \bar{K})$ is the kernel of the map of curve classes, $\operatorname{Hom}_{\mathbb{Z}}\left(\operatorname{Pic}\left(X_{\bar{K}}\right), \mathbb{Z}\right) \rightarrow \operatorname{Hom}_{\mathbb{Z}}\left(\operatorname{Pic}\left(C_{\bar{K}}\right), \mathbb{Z}\right)$.

(v) For every $f_{K}$-ample divisor class $H$ on the generic fiber $Y$ of $f_{K}$, for the associated complete intersection curve class $\theta=(H \cdots H)_{Y}$, the extension of smooth group $K$-schemes,

$$
0 \rightarrow\left|B Q_{\frac{C_{K}}{K}}^{0}\right| \rightarrow\left|B Q_{\frac{C_{K}}{K}}\right| \rightarrow \Lambda\left(Q / C_{K} / K\right) \rightarrow 0,
$$

has split restriction on the infinite subgroup $\mathbb{Z} \cdot \theta \subset \Lambda\left(Q / C_{K} / K\right)(K)$. 
(vi) For every universal $f_{K}$-torsor $\mathcal{T}$ on $X_{K}$, there is an associated $K$-morphism

$$
\alpha_{\mathcal{T}}: \operatorname{Hilb}_{\frac{X_{K}}{K}, \mathrm{CM}} \rightarrow\left|B Q_{C_{K} / K}\right|,
$$

whose restriction to $\operatorname{Sec}\left(X_{K} / C_{K} / K\right)$ sends each section $\sigma$ to $\sigma^{*} \mathcal{T}$ (see [Zhu19, Proposition 3.3]).

Even without the universal torsor $\mathcal{T}$, there is still a natural morphism $\alpha$ defined on $\mathrm{Hilb}_{X_{K} / K, \mathrm{CM}}$ mapping every connected component to a torsor (over some finite extension of Spec $K$ ) for $\left|B Q_{C_{K} / K}^{0}\right|$. This is the Abel torsor. Since every pseudosection in the connected component maps to a $K$-point of the Abel torsor, there exists a pseudosection only if the Abel torsor is a trivial torsor for $\left|B Q_{C_{K} / K}^{0}\right|$ over $\operatorname{Spec} K$. The construction of $\alpha_{\mathcal{T}}$ using a universal torsor shows that, for every $e$ in the infinite subgroup $\mathbb{Z} \cdot \theta$, the Abel torsor $\left|B Q_{C_{K} / K}^{e}\right|$ is actually a trivial torsor, i.e., it has $K$-points $I$. Because $K$ is the fraction field of a complete DVR, if there exists at least one $K$-point $I$, then every dense, Zariski open subset of $\left|B Q_{C_{K} / K}^{e}\right|$ contains a Zariski dense set of $K$-points.

\subsection{Smoothness of the Abel maps.}

Denote by $g$ the genus of the smooth, projective, geometrically connected $K$-curve $C_{K}$. For every algebraically closed extension $k / K$, a $k$-morphism $\sigma: C_{k} \rightarrow X_{k}$ is $(g)$-free if the image is contained in the smooth locus of $f_{k}$ and for every invertible sheaf $\mathcal{L}$ on $C_{k}$ of degree $d \leqslant \max (2 g, 0)$, the locally free sheaf $\operatorname{Hom}_{\mathcal{O}_{C_{k}}}\left(\sigma^{*} \Omega_{f_{k}}, \mathcal{L}\right)$ has vanishing $h^{1}$. There is an open subscheme $\operatorname{Sec}\left(X_{K} / C_{K} / K\right)_{\text {free }}$ of $\operatorname{Sec}\left(X_{K} / C_{K} / K\right)$ such that for every $k / K$, the $k$-points of $\operatorname{Sec}\left(X_{K} / C_{K} / K\right)_{\text {free }}(k)$ are precisely the $(g)$-free sections. For every $(g)$-free section $\sigma$, for every closed $k$-curve $\widetilde{C}$ in $X_{k}$ obtained by gluing to Image $\left(\sigma_{k}\right)$ at $\delta$ distinct $k$-points a free, genus 0 curve in the smooth locus of the corresponding fiber of $f_{k}$, the point $[\widetilde{C}]$ of the Hilbert scheme is a smooth point of $\Sigma\left(X_{R} / C_{R} / R\right)($ Spec $k)$, and all formal deformations of $[\widetilde{C}]$ to sections parameterized by $\operatorname{Sec}\left(X_{R} / C_{R} / R\right)(\operatorname{Spec} k((r)))$ are also $(g)$-free sections. Finally, the Abel map is smooth at $[\widetilde{C}]$ under a weak hypothesis. Let $C_{K}^{\prime} \rightarrow C_{K}$ be a quasi-finite, étale morphism such that $P \times_{C_{K}} C_{K}^{\prime}$ is trivial, $\mathbb{Z}^{\rho}$. For each glued free rational curve, choose a lift of the attaching point to $C_{K}^{\prime}$ so that the curve class is well defined in the constant group scheme $\operatorname{Hom}_{\mathbb{Z}}\left(P \times_{C_{K}} C_{K}^{\prime}, \mathbb{Z}\right)$ with fiber $N_{1} \cong \mathbb{Z}^{\rho}$. The Abel map is smooth at $[\widetilde{C}]$ if there are at least $(2 g-2) \rho$ free curves that can be partitioned into $\geqslant 2 g-2$ subsets of $\rho$ free rational curves whose classes in $N_{1} \otimes_{\mathbb{Z}} \mathbb{Q} \cong \mathbb{Q}^{\rho}$ span as a $\mathbb{Q}$-vector space.

\subsection{Abel sequences.}

There is a "positive structure" on $\Lambda\left(Q / C_{K} / K\right)$. This is the unique étale semigroup subscheme $\Lambda^{+}\left(Q / C_{K} / K\right)$ of $\Lambda\left(Q / C_{K} / K\right)$ whose associated subsemigroup $\Lambda^{+}\left(Q / C_{K} / K\right)(\operatorname{Spec} \bar{K})$ equals the semigroup of "effective" curve classes, i.e., those curve classes having non-negative pairing with all $f_{\bar{K}}$-ample invertible sheaves on 
$X_{\bar{K}}$. If the Mori cone of the geometric generic fiber of $f_{K}$ is finitely generated, then also $\Lambda^{+}\left(Q / C_{K} / K\right)(\operatorname{Spec} \bar{K})$ is finitely generated: it equals the invariant subsemigroup of the Mori cone for the (finite) action of the Galois group of $\bar{K}\left(C_{\bar{K}}\right)$. For every $e_{0} \in \Lambda^{+}\left(Q / C_{K} / K\right)(\operatorname{Spec} K)$, denote by $\Lambda^{\geqslant e_{0}}\left(Q / C_{K} / K\right)$ the translate of $\Lambda^{+}\left(Q / C_{K} / K\right)$ by $e_{0}$. Denote by $\Sigma \geqslant e_{0}\left(Q / C_{K} / K\right)$ the inverse image in $\Sigma\left(Q / C_{K} / K\right)$ of $\Lambda \geqslant e_{0}\left(Q / C_{K} / K\right)$.

Definition 2.5. - A sequence of $(g)$-free sections is an $K$-rational point $e_{0}$ of $\Lambda\left(Q / C_{K} / K\right)$ and a closed subscheme $Z_{\geqslant e_{0}}$ of $\Sigma \geqslant e_{0}\left(X_{K} / C_{K} / K\right)$

(i) whose open subscheme $Z_{\geqslant e_{0}} \cap \operatorname{Sec}\left(X_{K} / C_{K} / K\right)_{\text {free }}$ is dense,

(ii) whose fiber $Z_{e}$ over every geometric point e of $\Lambda^{\geqslant e_{0}}\left(Q / C_{K} / K\right)$ is an irreducible component of $\Sigma^{e}\left(X_{K} / C_{K} / K\right)$, and

(iii) for every geometric point $[\sigma]$ of $\operatorname{Sec}\left(X_{K} / C_{K} / K\right)_{\text {free, }}$, for all $\delta \geqslant \delta_{0}$, the scheme $Z_{\geqslant e_{0}}$ parameterizes every curve $\widetilde{C}$ obtained by gluing to Image $(\sigma)$ at $\delta$ distinct points a free, genus 0 curve in the smooth locus of the fiber of $f_{K}$.

The sequence is a pseudo Abel sequence, resp. an Abel sequence, if for the morphism,

$$
\alpha_{\mathcal{T}_{K}, Z_{e}}: Z_{\geqslant e_{0}} \cap \operatorname{Sec}\left(X_{K} / C_{K} / K\right) \rightarrow\left|B Q_{C_{K} / K}^{\geqslant e_{0}}\right|,
$$

every geometric generic fiber is nonempty and integral, resp. "birationally rationally connected" (every projective model is rationally connected). Define $Z_{R, \geqslant e_{0}}$ to be the closure of $Z_{\geqslant e_{0}}$ in $\Sigma\left(X_{R} / C_{R} / R\right)$.

Proposition 2.6. - For every projective $\mathbb{F}(\eta)$-scheme $X_{\eta}$, for every projective extension over $R$ of $X_{\eta}$ whose $K$-fiber $f_{K}: X_{K} \rightarrow C_{K}$ is a Picard-free morphism admitting a universal torsor $\mathcal{T}$ (i.e., the elementary obstruction vanishes), if there exists an Abel sequence $Z_{\geqslant e_{0}}$, then for every $K$-point $e$ of $\Lambda\left(Q / C_{K} / K\right)$ and for every general $K$-point $I$ of $\left|B Q_{C_{K} / K}^{e}\right|$, there exists an $\mathbb{F}(\eta)$-point of $X_{\eta}$, a finite flat extension of DVRs $R \rightarrow R^{\prime}$ with fraction field extension $K \rightarrow K^{\prime}$, and a $K^{\prime}$ point $\left[\sigma_{K^{\prime}}\right] \in Z_{e}$ whose image under $\alpha_{\mathcal{T}}$ equals the base change of $I$ and whose specialization over $\eta \times_{\text {Spec } R}$ Spec $R^{\prime}$ equals the base change of the $\mathbb{F}(\eta)$-point of $X_{\eta}$.

Proof. - Since $K$ has characteristic 0, by [Hir64], there exists a projective, birational morphism $\nu: \widetilde{Z}_{\geqslant e_{0}} \rightarrow Z_{\geqslant e_{0}}$ such that $\widetilde{Z}_{\geqslant e_{0}}$ is $K$-smooth. Moreover, there exists such $\nu$ for which the Abel map $\alpha_{\mathcal{T}}$ extends to a projective morphism,

$$
\widetilde{\alpha}_{\mathcal{T}}: \widetilde{Z}_{\geqslant e_{0}} \rightarrow\left|B Q_{C_{K} / K}^{\geqslant e_{0}}\right| \text {. }
$$

By generic smoothness, there exists a dense open subscheme of $\left|B Q_{C_{K} / K}^{\geqslant e_{0}}\right|$ over which $\widetilde{\alpha}_{\mathcal{T}}$ is smooth. Over this open subscheme, since every geometric generic fiber is rationally connected, every fiber is rationally connected. For $I$ in this open subscheme, denote by $\widetilde{Z}_{K, I}$ the fiber of $\widetilde{\alpha}_{\mathcal{T}}$ over $I$, and denote by $\nu_{K}: \widetilde{Z}_{K, I} \rightarrow \Sigma\left(X_{K} / C_{K} / K\right)$ the restriction of $\nu$. This is a smooth pseudosection of $\operatorname{Sec}\left(X_{K} / C_{K} / K\right)$. Now apply Proposition 2.2.

Proof of Theorem 1.4. - By hypothesis, $f_{K}$ is either a rationally simply connected fibration of Picard rank 1 as in [dJHS11, Theorem 13.1], or a rationally simply connected fibration as in [Zhu19, 5.1]. Then by [dJHS11, Theorem 13.1], resp. by [Zhu19, Theorem 5.12], there exists an Abel sequence. By Remark 2.4(v), for every $f_{K}$-ample 
divisor class $H$ on the generic fiber of $f_{K}$, for $\theta=(H \cdots H)$, for every integer $d \gg 0$, the class $e=d \cdot \theta$ gives a $K$-point of $\Lambda^{\geqslant e_{0}}\left(Q / C_{K} / K\right)$ such that $\left|B Q_{C_{K} / K}^{e}\right|$ has an $K$-point. For every general $K$-point $I$ of $\left|B Q_{C_{K} / K}^{e}\right|$, by Proposition 2.6, there exists an $\mathbb{F}(\eta)$-point of $X_{\eta}$ whose base change over $\eta \times{ }_{\text {Spec } R}$ Spec $R^{\prime}$ equals the specialization of a section $\sigma_{K^{\prime}}$ whose Abel image equals the base change of $I$.

\section{Projective Extensions}

For each of the four corollaries, we explain how to find projective extensions whose generic fiber is a rationally simply connected fibration over a curve. Combined with Theorem 1.4, this produces rational points.

\subsection{Projective Homogeneous Spaces. Corollaries 1.7 and 1.8.}

Definition 3.1. - For a stack $\mathcal{M}$ over a scheme $S$, a generic splitting variety of $\mathcal{M}$ (ala Amitsur) is a pair $\left(M, \zeta_{M}\right)$ of a locally finitely presented $S$-scheme $M$ and a 1-morphism over $S$,

$$
\zeta_{M}: M \rightarrow \mathcal{M}
$$

such that for every field $E$ and for every 1-morphism, $\zeta:$ Spec $E \rightarrow \mathcal{M}$, there exists a morphism $z: \operatorname{Spec} E \rightarrow M$ with $\zeta_{M} \circ z$ equivalent to $\zeta$.

For every fppf group $S$-scheme $G_{S}$, the classifying stack $B G_{S}$ is the stack over the fppf site of $S$-schemes whose fiber category over an $S$-scheme $T$ has as objects the $G_{S}$-torsors over $T$ and has as morphisms the $G_{S^{-}}$equivariant $T$-morphisms of torsors [LMB00, Proposition 10.13.1]. A smooth, affine group scheme $G_{S, 0}$ over $S$ is a reductive group scheme if the geometric fibers are connected and (geometrically) reductive, i.e., the unipotent radical is trivial. A flat, affine group scheme $G_{S}$ over $S$ is finite-by-reductive if there exists a surjective, smooth morphism of group $S$-schemes

$$
G_{S} \rightarrow \pi_{0} G_{S}
$$

where $\pi_{0} G_{S}$ is a finite, flat group $S$-scheme and the kernel, $G_{S, 0}$, is a reductive group $S$-scheme.

TheOrem 3.2. - [SdJ10] [dJHS11, Section 16] Let $R$ be a DVR, and let $S$ be Spec $R$ in the above setup. For every "finite-by-reductive" group $R$-scheme, $G_{R}$, for every integer $c \geqslant 1$, there exists a projective, fppf $R$-scheme $\bar{M}$, an open subscheme $M$ whose closed complement $\partial \bar{M}$ has codimension $>c$ in every fiber $\bar{M}_{s}$, and a 1-morphism $\zeta_{M}: M \rightarrow B G_{R}$ that is a generic splitting variety.

Proof. - There exists a linear representation of $G_{R}$ on a finite free $R$-module $V$ whose induced action on $\mathbb{P}_{R} V$ is free on a dense open subset $\left(\mathbb{P}_{R} V\right)^{o}$ whose complement in the semistable locus $\left(\mathbb{P}_{R} V\right)^{\mathrm{ss}}$ has codimension $>c$ in every fiber over Spec $R$. By [Ses77], the uniform categorical quotient of the action of $G_{R}$ on $\left(\mathbb{P}_{R} V\right)^{\mathrm{ss}}$ is a projective $R$-scheme $\bar{M}$, and there exists a unique open subscheme $M$ such that $\left(\mathbb{P}_{R} V\right)^{o}$ is a $G_{R^{-}}$torsor over $M$. This defines $\zeta_{M}: M \rightarrow B G_{R}$. 
By construction, $\bar{M}$ is normal and integral. Since it is also projective over the DVR $R$, and since it dominates the generic point Spec $K, \bar{M}$ is $R$-flat. Since the complement of $\left(\mathbb{P}_{R} V\right)^{o}$ has codimension $>c$, also $\partial \bar{M}$ has codimension $>c$. Since $\left(\mathbb{P}_{R} V\right)^{\circ}$ is $R$-smooth, and since $\left(\mathbb{P}_{R} V\right)^{\circ} \rightarrow M$ is flat, also $M$ is $R$-smooth, [Gro65, Proposition 17.7.7].

For every $\zeta$ : Spec $E \rightarrow B G_{R}$, the 2-fibered product $M \times_{\zeta_{M}, \zeta}$ Spec $E$ with its projection to Spec $E$ is $\left(\mathbb{P}_{E} V_{\zeta}\right)^{o}$ where $V_{\zeta}$ is the $E$-vector space $\operatorname{Hom}_{E}\left(E^{\oplus c+1}, \operatorname{Hom}_{E}\left(W_{0}, W\right)\right)$ for finite free $E$-vector spaces $W_{0}, W$ of equal (positive) rank. The free locus contains the locus parameterizing $(c+1)$-tuples $\left(\lambda_{0}, \ldots, \lambda_{c}\right)$ of $E$-linear maps $\lambda_{i}: W_{0} \rightarrow W$ that are isomorphisms. This locus has $E$-rational points. Thus, there exists an $R$ morphism $\lambda$ : Spec $E \rightarrow M$ and a 2-equivalence of the composition $\zeta_{M} \circ \lambda$ with $\zeta$.

Let $H_{S}$ be a split, connected and simply connected, semisimple group scheme over $S$. Let $P_{S} \hookrightarrow H_{S}$ be a standard parabolic subgroup scheme (containing a specified Borel). This has a Levi decomposition. Denote by $\chi: P_{S} \rightarrow Q_{S}$ the multiplicative quotient, i.e., the quotient of the Levi factor $L\left(P_{S}\right)=P_{S} / \operatorname{rad}^{u}\left(P_{S}\right)$ by its commutator subgroup $S$-scheme $L^{(1)}\left(P_{S}\right)$. Consider the left regular action of $P_{S}$ on $H_{S}, p \cdot h=p h$. Consider the diagonal left action of $P_{S}$ on $Q_{S} \times_{S} H_{S}, p \cdot(q, h)=\left(q \chi(p)^{-1}, p h\right)$. The projection morphism pr $2: Q_{S} \times_{S} H_{S} \rightarrow H_{S}$ is $P_{S^{-}}$equivariant. Finally, the left regular $Q_{S^{-}}$-action,

$$
Q_{S} \times_{S}\left(Q_{S} \times_{S} H_{S}\right) \rightarrow Q_{S} \times_{S} H_{S}, q^{\prime} \cdot(q, h)=\left(q^{\prime} q, h\right),
$$

is $P_{S^{-}}$-equivariant. By fppf descent, there are affine morphisms

$$
H_{S} \rightarrow P_{S} \backslash H_{S}, \quad Q_{S} \times{ }_{S} H_{S} \rightarrow P_{S} \backslash\left(Q_{S} \times{ }_{S} H_{S}\right)
$$

that are $P_{S^{-}}$torsors. The $P_{S^{-}}$-equivariant morphisms above induce morphisms

$$
\begin{gathered}
\operatorname{pr} 2: P_{S} \backslash\left(Q_{S} \times{ }_{S} H_{S}\right) \rightarrow P_{S} \backslash H_{S}, \\
Q_{S} \times{ }_{S} P_{S} \backslash\left(Q_{S} \times{ }_{S} H_{S}\right) \rightarrow P_{S} \backslash\left(Q_{S} \times{ }_{S} H_{S}\right) .
\end{gathered}
$$

Moreover, the scheme $P_{S} \backslash H_{S}$ is projective over $S$ : the dual of the relative dualizing sheaf is relatively very ample, cf. [Dem77, p. 186]. The other schemes are affine over $P_{S} \backslash H_{S}$, hence can be constructed by fpqc descent for affine schemes. Altogether these quotients and morphisms make $\mathcal{T}_{S}:=P_{S} \backslash\left(Q_{S} \times_{S} H_{S}\right)$ into a (left) $Q_{S}$-torsor over $P_{S} \backslash H_{S}$.

Definition 3.3. - For the pair $\left(H_{S}, P_{S}\right)$ as above, the associated projective homogeneous scheme is $X_{H, P}=P_{S} \backslash H_{S}$.

Lemma 3.4. - [Zhu19, Construction 5.3], [BCTS08, Lemma 2.2(vi)], [Gil10, Lemma 6.4] The Picard torus of $X_{H, P}$ over $S$ is $Q_{S}$ and the Picard lattice is the character lattice $Q_{S}^{D}$. The $Q_{S}$-torsor $\mathcal{T}_{S}$ is a universal torsor on $P_{S} \backslash H_{S}$.

Since $H_{S} \rightarrow X_{H, P}$ is a $P_{S}$-torus, since $P_{S}$ is $S$-flat, and since $H_{S}$ is $S$-smooth, $X_{H, P}$ is $S$-smooth, [Gro65, Proposition 17.7.7].

Notation 3.5. - Denote by $\rho_{X}$ the unique morphism of group $S$-schemes,

$$
\rho_{X}: Q_{S} \rightarrow \mathbb{G}_{m, S},
$$

such that the associated $\mathbb{G}_{m, S}$-torsor $\rho_{X, *} \mathcal{T}_{S}$ is the $\mathbb{G}_{m, S}$-torsor of the very ample invertible sheaf that is the dual of the relative dualizing sheaf. 
The automorphism group scheme of the pair $\left(X_{H, P}, \omega_{X / S}^{\vee}\right)$ is an affine group $S$ scheme, cf. [SdJ10, Section 2.1]. This is essentially just [Gro62, No. 221, Section 4.c], [Gro63, Corollaire 7.7.8], [LMB00, Théorème 4.6.2.1]. The same method proves that the automorphism group scheme $G_{S}^{\prime}$ of the pair $\left(X_{H, P}, \mathcal{T}_{S}\right)$ is an affine group $S$ scheme. Since $\mathcal{T}_{S}$ is intrinsic, the forgetful morphism

$$
\operatorname{Aut}_{S}\left(X_{H, P}, \mathcal{T}_{S}\right) \rightarrow \operatorname{Aut}_{S}\left(X_{H, P}\right)
$$

is surjective. The domain group scheme, $G_{S}^{\prime}$, is smooth, and the identity component of the target is a semisimple group $S$-scheme, [Dem77, Proposition 4]. The kernel of the forgetful morphism is $Q_{S}$ with its induced action on $\mathcal{T}_{S}$. Thus, $G_{S}^{\prime}$ is a smooth, affine group $S$-scheme whose identity component $G_{S, 0}$ is a reductive group scheme.

The quotient $G_{S}^{\prime} / G_{S, 0}$ is a quasi-finite, étale group scheme $\pi_{0} G_{S}^{\prime}$ over $S$. When $S$ is Spec $R$ for a Henselian local ring, there is a unique closed subgroup scheme $\pi_{0} G_{S \text {,fin }}^{\prime}$ of $\pi_{0} G_{S}^{\prime}$ that is finite, étale over $S$ and whose closed fiber $\pi_{0} G_{F, \text { fin }}^{\prime}$ equals the closed fiber $\pi_{0} G_{F}^{\prime}$.

By construction $G_{S}$ is finite-by-reductive. Denote the induced action of $G_{S}^{\prime}$, as a right action

$$
\begin{aligned}
& \gamma_{X}: X_{H, P} \times_{S} G_{S}^{\prime} \rightarrow X_{H, P}, \quad\left(P_{S} \cdot h, g\right) \mapsto\left(P_{S} \cdot h\right) \bullet g . \\
& \gamma_{\mathcal{T}}: \mathcal{T}_{S} \times{ }_{S} G_{S}^{\prime} \rightarrow \mathcal{T}_{S} .
\end{aligned}
$$

Let $G_{S} \subset G_{S}^{\prime}$ be a closed subgroup scheme that contains $G_{S, 0}$ and that is finiteby-reductive, i.e., $G_{S, 0}$ is the kernel of a smooth, surjective group homomorphism $G_{S} \rightarrow \pi_{0} G_{S}$ with $\pi_{0} G_{S}$ finite and flat. The action of $G_{S}$ on $X_{H, P}$ induces an action on the Picard lattice $Q_{S}^{D}$. This action factors through an action of $\pi_{0} G_{S}$ on $Q_{S}^{D}$.

Definition 3.6. - The triple $\left(H_{S}, P_{S}, G_{S}\right)$ of a split, simply connected, semisimple group $S$-scheme $H_{S}$, a standard parabolic $P_{S}$, and a finite-by-reductive subgroup $S$-scheme of $\operatorname{Aut}_{S}\left(X_{H, P}, \mathcal{T}_{S}\right)$ containing the identity component is primitive if the induced action of $\pi_{0} G_{S}$ on the Picard lattice $Q_{S}^{D}$ of $P_{S} \backslash H_{S}$ has a rank 1 invariant sublattice.

Since it is intrinsic, one nonzero element in the invariant lattice is the character $\rho_{X}$ giving rise to the ample invertible sheaf $\omega_{X / S}^{\vee}$. Thus, the triple is primitive if and only if every invariant character is commensurate with $\rho_{X}$.

For every $S$-scheme $T$ and for every left $G_{S^{-}}$torsor $E_{T}$ over $T$,

$$
(\mu, \operatorname{pr} 2): G_{S} \times_{S} E_{T} \cong E_{T} \times_{T} E_{T}, \quad(g, x) \mapsto(\mu(g, x), x),
$$

there are induced left actions of $G_{S}$ as follows,

$$
\begin{array}{rlrl}
\tilde{\mu}_{X}: G_{S} \times_{S}\left(X_{H, P} \times_{S} E_{T}\right) \rightarrow X_{H, P} \times_{S} E_{T}, & (g,(x, y)) & \mapsto\left(x \gamma_{X}\left(g^{-1}\right), \mu(g) y\right), \\
\tilde{\mu}_{\mathcal{T}}: G_{S} \times{ }_{S} \mathcal{T}_{S} \times_{S} E_{T} \rightarrow \mathcal{T}_{S} \times_{S} E_{T}, & (g,(q, y)) \mapsto\left(q \gamma_{\mathcal{T}}\left(g^{-1}\right), \mu(g) y\right) .
\end{array}
$$

These are free action. Associated to the $G_{S}$-invariant cocharacter $\rho_{X}$, the action $\tilde{\mu}_{\mathcal{T}}$ induces a $G_{S}$-linearization over $X_{H, P} \times_{S} E_{T}$ of the relatively ample invertible sheaf pr $1^{*} \omega_{X / S}^{\vee}$. Thus, by fppf descent in the projective case, there is an affine $T$-morphism that is a $G_{S^{-}}$torsor,

$$
q_{X, E}: X_{H, P} \times_{S} E_{T} \rightarrow X_{E},
$$


together with a $G_{S^{-}}$torsor,

$$
q_{\mathcal{T}, E}: \mathcal{T}_{S} \times_{S} E_{T} \rightarrow \mathcal{T}_{E},
$$

and a quotient morphism

$$
\mathcal{T}_{E} \rightarrow X_{E}
$$

that is a $Q_{S}$-torsor.

Definition 3.7. - The pair $\left(X_{E}, \mathcal{T}_{E}\right)$ is the E-twist of $\left(X_{H, P}, \mathcal{T}_{S}\right)$.

Notation 3.8. - For a triple $\left(H_{S}, P_{S}, G_{S}\right)$, let $\left(\bar{M}, \mathcal{O}_{\bar{M}}(1), i: M \rightarrow \bar{M}\right)$ be a datum with codimension $>1$ boundary as in Theorem 3.2. For the 1-morphism $\zeta_{M}: M \rightarrow B G_{S}$, denote by $f_{M}: X_{M} \rightarrow M$ and $\mathcal{T}_{M}$ the associated twist of $\left(X_{H, P}, \mathcal{T}_{S}\right)$.

Hypothesis 3.9. - With notation as in Section 1, let $X_{\eta}$ be an $\mathbb{F}(\eta)$-scheme that is projective homogeneous. Assume that $X_{\eta}$ has a universal torsor $\mathcal{T}_{\eta}$.

There exists a pair $\left(H_{S}, P_{S}\right)$ as above such that $X_{H, P} \times_{\text {Spec } R} \operatorname{Spec} \mathbb{F}(\eta)$ is geometrically isomorphic to $X_{\eta}$. Let $Q_{\eta}$ be the Picard torus of $X_{\eta}$.

Notation 3.10. - Denote by $G_{S} \subset \operatorname{Aut}_{S}\left(X_{H, P}, \mathcal{T}_{H, P}\right)$ the unique closed subgroup scheme that is finite-by-reductive, that contains the identity component, and such that $\pi_{0} G_{\mathbb{F}}$ is the group of connected components of $\operatorname{Aut}_{\mathbb{F}}\left(X_{H, P}, \mathcal{T}_{H, P}\right)$.

The Isom scheme from $\left(X_{\eta}, \mathcal{T}_{\eta}\right)$ to $\left(X_{H, P}, \mathcal{T}_{H, P}\right)$ is a left $G_{S^{-}}$torsor over $\mathbb{F}(\eta)$. The $S$-smooth scheme $M$ from Theorem 3.2, for $c=1$, is a generic splitting variety. Thus, there is an induced morphism $\zeta: C_{\mathbb{F}} \rightarrow \bar{M}$. If necessary, replace $\bar{M}$ by $\bar{M} \times{ }_{S} \mathbb{P}_{S}^{3}$ so that $\zeta$ is a closed immersion. Denote the relative dimension of $\bar{M}$ by $m$. Denote by $\left(X_{M}, \mathcal{T}_{M}\right)$ the twist of $\left(X_{H, P}, \mathcal{T}_{H, P}\right)$ by the torsor $\zeta_{M}: M \rightarrow B G_{S}$.

Theorem 3.11. - [Zhu19] Assume Hypothesis 3.9. For every general $(m-1)$ tuple $\left(D_{1}, \ldots, D_{m-1}\right)$ of very ample divisors of $\bar{M}$ that contain $\zeta\left(C_{\mathbb{F}}\right)$, the common intersection $C_{R}=D_{1} \cap \ldots \cap D_{m-1}$ gives a projective extension of $X_{\eta} \rightarrow \operatorname{Spec} \mathbb{F}(\eta)$. If $\left(H_{S}, P_{S}, G_{S}\right)$ is primitive, then $X_{K} \rightarrow C_{K}$ and $\mathcal{T}_{K}$ satisfies the hypotheses of [Zhu19, 5.1], and the family has an Abel sequence.

Proof. - Since $C_{\mathbb{F}}$ is smooth, and since the image of $\zeta$ intersects the smooth open subscheme $M$, there exist very ample divisors $\left(D_{1, \mathbb{F}}, \ldots, D_{m-1, \mathbb{F}}\right)$ that contain $\zeta\left(C_{\mathbb{F}}\right)$ and whose common intersection $C_{\mathbb{F}}^{\prime}$ is a curve containing a dense open subset of $\zeta\left(C_{\mathbb{F}}\right)$ as an open subset. Since the boundary $\partial \bar{M}$ has codimension $>1$ in $\bar{M}$, for a general lift $\left(D_{1}, \ldots, D_{m-1}\right)$ (inside the complete linear system $\mathbb{P}_{R}^{N}$ over the Henselian ring $R$ ), the curve $C_{K}$ is a smooth curve that is contained in $M$. Thus, the restriction of $\left(X_{M}, \mathcal{T}_{M}\right)$ gives a projective extension of $\left(X_{\eta}, \mathcal{T}_{\eta}\right)$.

By the Bertini irreducibility theorem, for a general complete intersection curve $C_{K}$, the sections of the relative Picard of $X_{M} / M$ over $C_{K}$ equal the sections over all of $M_{K}$. If $\left(H_{S}, P_{S}, G_{S}\right)$ is primitive, the group of sections is a free Abelian group of rank 1. Thus, the restricted family $X_{K} \rightarrow C_{K}$ with the restricted universal torsor $\mathcal{T}_{\eta}$ satisfies [Zhu19, 5.1]. By the proof of [Zhu19, Theorem 1.4], this family has an Abel sequence. 
Proofs of Corollaries 1.7 and 1.8. - For Corollary 1.7, let $A$ be a division algebra with center $\mathbb{F}(\eta)$ that has finite dimension $n^{2}$ over the center and whose order in the Brauer group of $\mathbb{F}(\eta)$ equals $\ell$. Then $\ell$ divides $n$; the goal is to prove that $\ell$ equals $n$. Let $X_{\eta}$ be the generalized Severi-Brauer variety associated to $A$ that represents the functor on $\mathbb{F}(\eta)$-scheme associating to each $\mathbb{F}(\eta)$-scheme $T$ the set of local direct summands of $A \otimes_{\mathbb{F}(\eta)} \mathcal{O}_{T}$ of rank $\ell n$ over $\mathcal{O}_{T}$ and that are left ideals. Since the order of the Brauer element equals $\ell$, there exists a universal torsor $\mathcal{T}_{\eta}$ on $X_{\eta}$. Since $X_{\eta} \times_{\operatorname{Spec} \mathbb{F}(\eta)} \operatorname{Spec} \overline{\mathbb{F}(\eta)}$ is isomorphic to a Grassmannian, which has Picard group isomorphic to $\mathbb{Z}$, the scheme $X_{\eta}$ is primitive. By Theorem 3.11, there exists a projective extension that admits an Abel sequence. Thus, by Theorem 1.4, $X_{\eta}$ has an $\mathbb{F}(\eta)$-point, i.e., $A$ has an ideal of rank $\ell n$. The only left ideals in a division algebra are the zero ideal and all of $A$. Thus, $\ell n$ equals the dimension $n^{2}$ of $A$, i.e., $\ell$ equals $n$.

For Corollary 1.8, let $E$ be a left $G$-torsor over $\operatorname{Spec} \mathbb{F}(\eta)$. Since $G$ is quasi-split, there exists a Borel $B \subseteq G$. Let $X_{\eta}$ and $\mathcal{T}_{\eta}$ be the $E$-twist of $\left(X_{G, B}, \mathcal{T}_{\mathbb{F}(\eta)}\right)$. This might not be primitive. However, the inductive strategy of [Zhu19, Lemma 12.1] reduces existence of an $\mathbb{F}(\eta)$-point of $X_{\eta}$ to existence of an $\mathbb{F}(\eta)$-point of a projective homogeneous space $Y$ over $\mathbb{F}(\eta)$ whose elementary obstruction vanishes and that is primitive. This will imply that the generic fiber of the projective extension of $Y$ is also primitive. In the primitive case, by Theorem 3.11, there exists a projective extension that admits an Abel sequence. Thus, by Theorem 1.4, there is an $\mathbb{F}(\eta)$-point of $Y$.

\subsection{Complete Intersections. Corollary 1.6.}

Notation 3.12. - Let $S$ be a scheme. Let $n$ and $1 \leqslant b \leqslant n$ be positive integers. Let $\underline{d}=\left(d_{1}, \ldots, d_{b}\right)$ be an ordered $b$-tuple of integers $d_{i} \geqslant 2$. For each $j=1, \ldots, b$, denote by $V_{j}\left(d_{j}\right)$ the free $\mathcal{O}_{S}$-module $H^{0}\left(\mathbb{P}_{S}^{n}, \mathcal{O}_{\mathbb{P}_{S}^{r_{j}}}\left(d_{j}\right)\right)$. Denote by $V(\underline{d})$ the direct sum $V_{1}\left(d_{1}\right) \oplus \ldots \oplus V_{b}\left(d_{b}\right)$ as a free $\mathcal{O}_{S}$-module. Denote by $\mathbb{P}_{S} V(\underline{d})$ the projective space over $S$ on which there is a universal ordered $b$-tuple $\left(\phi_{1}, \ldots, \phi_{b}\right)$ of sections of the invertible sheaves $\mathcal{O}_{\mathbb{P}^{n}}\left(d_{j}\right)$. Precisely, for the product

$$
P=\mathbb{P}_{S} V(\underline{d}) \times_{S} \mathbb{P}_{S}^{n}
$$

with its projections

$$
\operatorname{pr}_{1}: P \rightarrow \mathbb{P}_{T} V(\underline{d}) \text { and } \operatorname{pr}_{2}: P \rightarrow \mathbb{P}_{T}^{n},
$$

the sequence $\left(\phi_{1}, \ldots, \phi_{b}\right)$ is a universal homomorphism of coherent sheaves

$$
\operatorname{pr}_{2}^{*} \mathcal{O}_{\mathbb{P}_{S}^{n}}\left(-d_{1}\right) \oplus \ldots \oplus \operatorname{pr}_{2}^{*} \mathcal{O}_{\mathbb{P}_{S}^{n}}\left(-d_{b}\right) \rightarrow \operatorname{pr}_{1}^{*} \mathcal{O}_{\mathbb{P}_{S} V(\underline{d})}(1),
$$

or equivalently, a universal homomorphism of coherent sheaves,

$$
\left(\phi_{1}, \ldots, \phi_{b}\right): \operatorname{pr}_{1}^{*} \mathcal{O}_{\mathbb{P}_{S} V(\underline{d})}(-1) \otimes\left(\operatorname{pr}_{2}^{*} \mathcal{O}_{\mathbb{P}_{T}^{n}}\left(-d_{1}\right) \oplus \ldots \oplus \operatorname{pr}_{2}^{*} \mathcal{O}_{\mathbb{P}_{T}^{n}}\left(-d_{b}\right)\right) \rightarrow \mathcal{O}_{P}
$$

For each $j=1, \ldots, b$, denote by $X_{j} \subset P$ the effective Cartier divisor defined by $\phi_{j}$. Denote by $X_{j}^{\prime}$ the intersection $X_{1} \cap \ldots \cap X_{j}$ as a closed subscheme of $P$. 
Definition 3.13. - For every $j=1, \ldots, b$, the smooth locus $\mathbb{P}_{S} V(\underline{d})_{j}^{\mathrm{sm}}$, resp. the Lefschetz locus $\mathbb{P}_{S} V(\underline{d})_{j}^{\text {Lef }}$, is the maximal open subscheme of $\mathbb{P}_{S} V(\underline{d})$ over which $X_{j}^{\prime}$ is flat and every geometric fiber is smooth, resp. has at worst a single ordinary double point. The degenerate locus, resp. the badly degenerate locus, is the closed complement of $\mathbb{P}_{S} V(\underline{d})_{j}^{\mathrm{sm}}$, resp. $\mathbb{P}_{S} V(\underline{d})_{j}^{\mathrm{Lef}}$.

Proposition 3.14. - [Del73, Exposé XVII, Théorème 2.5] Assume that $b<n$. Then for every $j=1, \ldots, b$, the degenerate locus is a proper closed subset, and the badly degenerate locus of $X_{j}^{\prime}$ is a proper closed subset of codimension $>1$.

Proof. - This is proved by induction on $j$. For $j=1$, this follows from loc. cit. By way of induction, assume that $j>1$ and assume that the result is proved for $j-1$. By loc. cit., the intersection with $\mathbb{P}_{S} V(\underline{d})_{j-1}^{\mathrm{sm}}$ of the degenerate locus, resp. badly degenerate locus, of $X_{j}^{\prime}$ is a proper closed subset, resp. has codimension $>1$. It suffices to prove that $\mathbb{P}_{S} V(\underline{d})_{j}^{\mathrm{sm}}$ contains every generic point $\xi$ of the degenerate locus of $X_{j-1}^{\prime}$ that is not in the badly degenerate locus. By hypothesis, $X_{j-1, \xi}^{\prime}$ has a unique ordinary double point. In $\mathbb{P}_{S} V(\underline{d})$, it is a codimension 1 condition for $\phi_{j}$ to vanish at this point. On the complement of this proper closed subset, the degenerate locus of $X_{j}^{\prime}$ is a proper closed subset by Bertini's smoothness theorem [Jou83, Theorème 6.3(2)].

Hypothesis 3.15. - Let $S$ be Spec $R$ for a Henselian DVR with finite residue field $\mathbb{F}$ and with characteristic 0 fraction field $K$. Let $\mathbb{F}(\eta)$ be $\mathbb{F}\left(C_{\mathbb{F}}^{\prime}\right)$ for a smooth, projective, geometrically connected $\mathbb{F}$-curve $C_{\mathbb{F}}^{\prime}$. Notations are as in Notation 3.12. Assume that $b<n$. Let $X_{\eta}^{\prime} \subset \mathbb{P}_{\mathbb{F}(\eta)}^{n}$ be an intersection $X_{\eta, 1} \cap \ldots \cap X_{\eta, b}$ of hypersurfaces $X_{\eta, i}=\operatorname{Zero}\left(\phi_{\eta, i}\right)$ of degree $d_{i}$. Denote by $\zeta: C_{\mathbb{F}}^{\prime} \rightarrow \mathbb{P}_{R} V(\underline{d})$ the $R$-morphism of $\left(\phi_{\eta, 1}, \ldots, \phi_{\eta, b}\right)$. Denote by $m$ the relative dimension of $\mathbb{P}_{R} V(\underline{d})$ over $\operatorname{Spec} R$.

TheOrem 3.16. - [DeL15, dJHS11] Assume Hypothesis 3.15. For every general $(m-1)$-tuple $\left(D_{1}, \ldots, D_{m-1}\right)$ of very ample divisors of $\mathbb{P}_{R} V(\underline{d})$ that contain $\zeta\left(C_{\mathbb{F}}^{\prime}\right)$, for the common intersection $C_{R}=D_{1} \cap \ldots \cap D_{m-1}$, for the restriction $X_{R}^{\prime}$ of $X_{b}^{\prime}$ over $C_{R}$, for the closure $X_{R}$ in $X_{R}^{\prime}$ of the generic fiber $X_{K, b}=X_{R}^{\prime} \times_{\operatorname{Spec} R} \operatorname{Spec} K$, the morphism $X_{R} \rightarrow C_{R}$ is a projective extension over $R$ of the fiber $X_{\eta} \rightarrow \operatorname{Spec} \mathbb{F}(\eta)$. Also, $X_{\eta}$ is a closed subscheme of $X_{\eta, b}^{\prime}$ that has pure dimension $n-b$. The restriction of the $\mathbb{G}_{m}$-torsor of $\mathcal{O}(1)$ on $\mathbb{P}_{R}^{n}$ is a universal torsor. If $d_{1}^{2}+\ldots+d_{b}^{2} \leqslant n$, then $X_{K, b} \rightarrow C_{K}$ is a rationally simply connected fibration (of Picard rank 1) in the sense of [dJHS11, Theorem 13.1], and the family has an Abel sequence.

Proof. - Existence of the projective extension is basically the same as in the proof of Theorem 3.11. By Proposition 3.14, for a general choice of $\left(D_{1}, \ldots, D_{m-1}\right)$, the curve $C_{K}$ is a smooth, projective, geometrically connected curve contained in the Lefschetz locus of $X_{b}$ and having dense intersection with the smooth locus.

Now assume that $d_{1}^{2}+\ldots+d_{b}^{2} \leqslant n$. There are three global hypotheses in [dJHS11, Theorem 13.1], and the remaining hypotheses are on the geometric generic fiber of $f_{K}: X_{K, b} \rightarrow C_{K}$. The first hypothesis is that $X_{K, b}$ is smooth. The projection $X_{b}^{\prime} \rightarrow \mathbb{P}_{R}^{n}$ is a projective space bundle, hence $X_{b}^{\prime}$ is $R$-smooth. By Bertini's theorem, [Jou83, Théorème 6.3(2)], for $D_{1}, \ldots, D_{m-1}$ general, the inverse image of $C_{K}=D_{K, 1} \cap \ldots \cap D_{K, m-1}$ under the projection $X_{b}^{\prime} \rightarrow \mathbb{P}_{R} V(\underline{d})$ is smooth.

The second hypothesis is that every geometric fiber of $f_{K}$ is irreducible. By hypothesis, every $d_{i} \geqslant 2$, so that $d_{1}^{2}+\ldots+d_{b}^{2} \geqslant 4 b$. Thus, $n \geqslant 4 b$, so that also $n-b \geqslant 3 b \geqslant 3$. 
Every fiber of $f_{K}$ is a complete intersection of ample divisors of dimension $\geqslant 3$. Thus, the complete intersection is connected, cf. [Gro03, Corollary XII.3.5]. Moreover, the complete intersection has at most a single ordinary double point. Thus, the fiber is normal by Serre's Criterion, cf. [Gro03, XI.3.11], hence it is irreducible.

The third global hypothesis is that $\mathcal{O}(1)$ is $f_{K}$-ample. In fact it is $f_{K}$-very ample since $X_{K}$ is a closed subscheme of $C_{K} \times \operatorname{Spec} K \mathbb{P}_{K}^{n}$.

The remaining hypotheses are all hypotheses of the geometric generic fiber of $f_{K}$, which is a smooth complete intersection with $d_{1}^{2}+\ldots+d_{b}^{2} \leqslant n$ that is general. These hypotheses are all established by Matt DeLand, [DeL15]. Therefore, by [dJHS11, Theorem 13.1], there exists an Abel sequence.

Proof of Corollary 1.6. By Theorem 3.16, there exists a projective extension with vanishing elementary obstruction and with an Abel sequence. By Theorem 1.4, there exists an $\mathbb{F}(\eta)$-point.

\subsection{Hypersurfaces in Grassmannians. Corollary 1.9.}

Notation 3.17. - Let $S$ be an affine scheme. Let $m>0$ be an integer, and let $\ell>0$ be an integer such that $m \geqslant 2 \ell$ (or else replace $\ell$ by $m-\ell$ ). Let $d>0$ be an integer. Let $H_{S}$ be $\mathbf{S L}_{m, S}$. Let $P_{S} \subset H_{S}$ be the parabolic that preserves the projection $\mathcal{O}_{S}^{\oplus m} \rightarrow \mathcal{O}_{S}^{\oplus \ell}$ onto the first $\ell$ factors. Thus, $X_{H, P}=P_{S} \backslash H_{S}$ is the Grassmannian $\operatorname{Grass}_{S}\left(\ell, \mathcal{O}_{S}^{\oplus m}\right)$. Also the universal torsor $\mathcal{T}_{H, P}$ is the $\mathbb{G}_{m}$-torsor associated to the Plücker invertible sheaf $\mathcal{O}(1)$. Let $G_{S}$ be $\operatorname{Aut}_{S}\left(X_{H, P}, \mathcal{T}_{H, P}\right)$. Let $W(d)$ be the free $\mathcal{O}_{S}$-module $H^{0}\left(X_{H, P}, \mathcal{O}(d)\right)$. Denote by $\mathbb{P}_{S} W(d)^{\text {Lef }}$ the Lefschetz locus parameterizing degree $d$ hypersurfaces that have at worst a single ordinary double point, cf. Definition 3.13.

Let $V_{S}$ be the linear representation of $G_{S}$ from the proof of Theorem 3.2 with $c=1$. Define $\bar{M}^{\prime}$ to be the uniform categorical quotient of the action of $G_{S}$ on the semistable locus of $\mathbb{P}_{S}\left(V_{S}\right) \times_{S} \mathbb{P}_{S} W(d)$. By the proofs of Theorem 3.2 and Proposition 3.14, there is an open subscheme $M^{\prime} \subset \bar{M}^{\prime}$ whose inverse image equals $\mathbb{P}_{S}\left(V_{S}\right)^{o} \times{ }_{S} \mathbb{P}_{S} W(d)^{\text {Lef }}$ and that has closed complement $\partial \bar{M}^{\prime}$ of codimension $>1$. Since $\mathbb{P}_{S}\left(V_{s}\right)^{o} \times{ }_{S} \mathbb{P}_{S} W(d)^{\text {Lef }} \rightarrow M^{\prime}$ is flat, also $M^{\prime}$ is $R$-smooth, [Gro65, Proposition 17.7.7]. Denote by $G_{M^{\prime}} \rightarrow M^{\prime}$ and $\mathcal{T}_{M^{\prime}}$ the twist of $X_{H, P}$ and $\mathcal{T}_{H, P}$. Denote by $X_{M^{\prime}} \subset G_{M^{\prime}}$ the universal degree $d$ hypersurface.

Hypothesis 3.18. - Let $S$ be Spec $R$ for a Henselian DVR with finite residue field $\mathbb{F}$ and with characteristic 0 fraction field $K$. Let $\mathbb{F}(\eta)$ be $\mathbb{F}\left(C_{\mathbb{F}}^{\prime}\right)$ for a smooth, projective, geometrically connected $\mathbb{F}$-curve $C_{\mathbb{F}}^{\prime}$. Let $G_{\eta}$ be a smooth, projective $\mathbb{F}(\eta)$ scheme that is geometrically isomorphic to $X_{H, P}$. Assume that there exists a universal torsor $\mathcal{T}_{\eta}$. Let $X_{\eta} \subset G_{\eta}$ be a closed subscheme that is geometrically isomorphic to a degree $d$ hypersurface in $X_{H, P}$. Denote by $\zeta: C_{\mathbb{F}}^{\prime} \rightarrow \bar{M}_{\mathbb{F}}^{\prime}$ the associated $R$-morphism. Denote by $s$ the relative dimension of $\bar{M}^{\prime}$ over $\operatorname{Spec} R$.

Theorem 3.19 ([dJHS11, Fin10]). — Assume Hypothesis 3.18. For every general $(s-1)$-tuple $\left(D_{1}, \ldots, D_{s-1}\right)$ of very ample divisors of $\bar{M}_{R}^{\prime}$ that contain $\zeta\left(C_{\mathbb{F}}^{\prime}\right)$, the 
common intersection $C_{R}=D_{1} \cap \ldots \cap D_{m-1}$ and the restriction $X_{R} \rightarrow C_{R}$ of $X_{M^{\prime}}$ gives a projective extension of $X_{\eta} \rightarrow \operatorname{Spec} \mathbb{F}(\eta)$. The restriction of $\mathcal{T}_{M^{\prime}}$ is a universal torsor. If $(3 \ell-1) d^{2}-d<m-4 \ell-1$, then $X_{K, b} \rightarrow C_{K}$ is a rationally simply connected fibration (of Picard rank 1) in the sense of [dJHS11, Theorem 13.1], and the family has an Abel sequence.

Proof. - The first part of the proof is precisely the same as in the proof of Theorem 3.16. The hypotheses on the geometric generic fiber of $f_{K}$ follow from [Fin10, Theorem 1.8].

Proof of Corollary 1.9. By Theorem 3.19, there exists a projective extension with vanishing elementary obstruction that has an Abel sequence. By Theorem 1.4, there exists an $\mathbb{F}(\eta)$-point.

\section{Acknowledgments}

We are very grateful to Aise Johan de Jong, in particular. We are grateful to both de Jong and Eduardo Esteves who pointed out that an Abel map as in [dJHS11] must exist under weaker hypotheses. The authors thank Yi Zhu for helpful discussions and Max Lieblich who explained the history of the Brauer-Hasse-Noether theorem. We are grateful to Jean-Louis Colliot-Thélène and the referees for their feedback on an early draft.

\section{BIBLIOGRAPHY}

[BCTS08] Mikhail Borovoi, Jean-Louis Colliot-Thélène, and Alexei N. Skorobogatov, The elementary obstruction and homogeneous spaces, Duke Math. J. 141 (2008), no. 2, 321-364. $\uparrow 1409$

[CTS87] Jean-Louis Colliot-Thélène and Jean-Jacques Sansuc, La descente sur les variétés rationnelles. II, Duke Math. J. 54 (1987), no. 2, 375-492. ^1400, 1402

[Del73] N. Deligne, Pierre et Katz, Groupes de monodromie en géométrie algébrique. II, Lecture Notes in Mathematics, vol. 340, Springer, 1973, Séminaire de Géométrie Algébrique du Bois-Marie 1967-1969 (SGA 7 II). $\uparrow 1413$

[DeL15] Matt DeLand, Relatively very free curves and rational simple connectedness, J. Reine Angew. Math. 699 (2015), 1-33. 11413,1414

[Dem77] Michel Demazure, Automorphismes et déformations des variétés de Borel, Invent. Math. 39 (1977), no. 2, 179-186. $\uparrow 1409,1410$

[DG70] Michel Demazure and Alexander Grothendieck, Schémas en groupes. II : Groupes de type multiplicatif, et structure des schémas en groupes généraux, Lecture Notes in Mathematics, vol. 152, Springer, 1970. $\uparrow 1405$

[dJ97] Aise Johan de Jong, Families of curves and alterations, Ann. Inst. Fourier 47 (1997), no. 2, 599-621. $\uparrow 1403$

[dJHS11] Aise Johan de Jong, Xehua He, and Jason M. Starr, Families of rationally simply connected varieties over surfaces and torsors for semisimple groups, Publ. Math., Inst. Hautes Étud. Sci. 114 (2011), no. 1, 1-85. 个1401, 1402, 1403, 1407, 1408, 1413, 1414, 1415

[Esn03] Hélène Esnault, Varieties over a finite field with trivial Chow group of 0-cycles have a rational point, Invent. Math. 151 (2003), no. 1, 187-191. $\uparrow 1403$ 
[Esn06] _ Deligne's integrality theorem in unequal characteristic and rational points over finite fields, Ann. Math. 164 (2006), no. 2, 715-730, With an appendix by Pierre Deligne and Esnault. $\uparrow 1403$

[Esn07] _ Coniveau over $\mathfrak{p}$-adic fields and points over finite fields, C. R. Math. Acad. Sci. Paris 345 (2007), no. 2, 73-76. $\uparrow 1400,1402,1403$

[EX09] Hélène Esnault and Chenyang Xu, Congruence for rational points over finite fields and coniveau over local fields, Trans. Am. Math. Soc. 361 (2009), no. 5, 2679-2688. ^1402, 1403

[Fin10] Robert Adam Findley, Rational curves in low degree hypersurfaces of Grassmannian varieties, Ph.D. thesis, State University of New York, Stony Brook, USA, 2010. ^1402, 1414,1415

[Fuj02] Kazuhiro Fujiwara, A proof of the absolute purity conjecture (after Gabber), Algebraic geometry 2000, Azumino (Hotaka), Advanced Studies in Pure Mathematics, vol. 36, Mathematical Society of Japan, 2002, pp. 153-183. $\uparrow 1403$

[GHMS05] Tom Graber, Joe Harris, Barry Mazur, and Jason Starr, Rational connectivity and sections of families over curves, Ann. Sci. Éc. Norm. Supér. 38 (2005), 671-692. $\uparrow 1404$

[Gil10] Philippe Gille, Serre's conjecture II: a survey, Quadratic forms, linear algebraic groups, and cohomology, Developments in Mathematics, vol. 18, Springer, 2010, pp. 41-56. $\uparrow 1409$

[Gre66] Marvin J. Greenberg, Rational points in Henselian discrete valuation rings, Publ. Math., Inst. Hautes Étud. Sci. (1966), no. 31, 563-568. $\uparrow 1400$

[Gro62] Alexander Grothendieck, Fondements de la géométrie algébrique. [Extraits du Séminaire Bourbaki, 1957-1962.], Secrétariat mathématique, 1962. ^1404, 1410

[Gro63] _ Éléments de géométrie algébrique. III. étude cohomologique des faisceaux cohérents. II, Publ. Math., Inst. Hautes Étud. Sci. 17 (1963), no. 1, 5-80. ^1410

[Gro65] _ Éléments de géométrie algébrique. IV. étude locale des schémas et des morphismes de schémas., Publ. Math., Inst. Hautes Étud. Sci. 24 (1965), no. 1, 5-223. $\uparrow 1409,1414$

[Gro03] _ Revêtements étales et groupe fondamental (SGA 1), Documents Mathématiques, vol. 3, Société Mathématique de France, 2003, Séminaire de géométrie algébrique du Bois Marie 1960-61. [Geometric Algebra Seminar of Bois Marie 1960-61]. Directed by A. Grothendieck, With two papers by M. Raynaud, Updated and annotated reprint of the 1971 original. $\uparrow 1414$

[Gro05] Cohomologie locale des faisceaux cohérents et théorèmes de Lefschetz locaux et globaux (SGA 2), Documents Mathématiques, vol. 4, Société Mathématique de France, 2005, Séminaire de Géométrie Algébrique du Bois Marie, 1962, Augmenté d'un exposé de Michèle Raynaud. [With an exposé by Michèle Raynaud], With a preface and edited by Yves Laszlo, Revised reprint of the 1968 French original. $\uparrow 1405$

[Har75] Günter Harder, Über die Galoiskohomologie halbeinfacher algebraischer Gruppen. III, J. Reine Angew. Math. 274/275 (1975), 125-138, Collection of articles dedicated to Helmut Hasse on his seventy-fifth birthday, III. $\uparrow 1402$

[Hir64] Heisuke Hironaka, Resolution of singularities of an algebraic variety over a field of characteristic zero. I, II, Ann. Math. 79 (1964), 109-203, 205-326. $\uparrow 1407$

[HX09] Amit Hogadi and Chenyang Xu, Degenerations of rationally connected varieties, Trans. Am. Math. Soc. 361 (2009), no. 7, 3931-3949. ^1402

[Jou77] Jean-Pierre Jouanolou, Cohomologie l-adique et fonctions L, Lecture Notes in Mathematics, vol. 589, Springer, 1977, Séminaire de Géometrie Algébrique du Bois-Marie 1965-1966 (SGA 5). $\uparrow 1404$ 
[Jou83] _ Théorèmes de Bertini et applications, Progress in Mathematics, vol. 42, Birkhäuser, 1983. $\uparrow 1413$

[Lan52] Serge Lang, On quasi algebraic closure, Ann. Math. 55 (1952), 373-390. $\uparrow 1402$

[Lie06] Max D. Lieblich, Remarks on the stack of coherent algebras, Int. Math. Res. Not. 2006 (2006), no. 11, 75273. $\uparrow 1405$

[LMB00] Gérard Laumon and Laurent Moret-Bailly, Champs algébriques, Ergebnisse der Mathematik und ihrer Grenzgebiete. 3. Folge., vol. 39, Springer, 2000. $\uparrow 1408,1410$

[Pir12] Alena Pirutka, R-équivalence sur les familles de variétés rationnelles et méthode de la descente, J. Théor. Nombres Bordeaux 24 (2012), no. 2, 461-473. $\uparrow 1400$

[Roq05] Peter Roquette, The Brauer-Hasse-Noether theorem in historical perspective, Schriften der Mathematisch-Naturwissenschaftlichen Klasse der Heidelberger Akademie der Wissenschaften, vol. 15, Springer, 2005. $\uparrow 1402$

[SdJ10] Jason M. Starr and Aise Johan de Jong, Almost proper GIT-stacks and discriminant avoidance, Doc. Math. 15 (2010), 957-972. $11403,1408,1410$

[Ses77] Conjeeveram S. Seshadri, Geometric reductivity over arbitrary base, Adv. Math. 26 (1977), no. 3, 225-274. $\uparrow 1408$

[Sko01] Alexei N. Skorobogatov, Torsors and rational points, Cambridge Tracts in Mathematics, vol. 144, Cambridge University Press, 2001. $\uparrow 1400$

[Zhu19] Yi Zhu, Homogeneous space fibrations over surfaces, J. Inst. Math. Jussieu 18 (2019), no. 2, 293-327. $\uparrow 1400,1401,1402,1405,1406,1407,1409,1411,1412$

Manuscript received on 27th March 2018,

revised on 7th August 2019,

accepted on 19th February 2020.

Recommended by Editor S. Cantat.

Published under license CC BY 4.0.

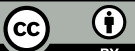

This journal is a member of Centre Mersenne.

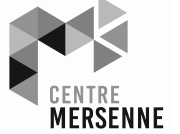

Jason STARR

Department of Mathematics,

Stony Brook University,

Stony Brook, NY 11794, (USA)

jstarr@math.stonybrook.edu

Chenyang XU

BICMR, Beijing, (China)

cyxu@math.pku.edu.cn 